\title{
Vascular Ehlers-Danlos syndrome, pixels, and high-definition clinical genomics
}

\author{
Michael F. Murray, MD'
}

High-definition television (HDTV), as compared with standard-definition television, delivers a higher-quality end product that can be appreciated by experts and nonexperts alike. HDTV was reportedly introduced about 25 years ago and had its inaugural public broadcast in $1996 .{ }^{1}$ Although I remain generally uninformed about the details of the long process behind the development of HDTV, I was not surprised to learn that since the first half of the 20th century there have been many dedicated professionals who have worked for years on the task of delivering to us the critical element of HDTV-more pixels. ${ }^{1}$ Pixels are defined as the "smallest controllable element of a picture displayed on a screen," and their number and density are the distinguishing features separating high definition (HD) from standard definition. ${ }^{2}$

Clinical genomics in this 21 st century has to arrive at a similar level of HD, whereby the nonexpert can easily appreciate the difference in quality between what we can offer now and a version that could be considered HD clinical genomics (HDCG). I suggest that, in HDCG, the equivalent of the pixel is the genotype-phenotype correlation at the level of the sequence variant. With the Human Genome Project complete, the work now is to add those essential details that will bring us to the day when the end user-for example, the patient-provider pair-can sit in a room and completely appreciate the quality of the HD product that has been produced through the dedicated work of many in the field.

Two articles on vascular Ehlers-Danlos syndrome (vEDS) appear in this issue of Genetics In Medicine: "Survival Is Affected by Mutation Type and Molecular Mechanism in Vascular Ehlers-Danlos Syndrome (EDS Type IV)," by Pepin and colleagues, ${ }^{3}$ and "Pregnancy-Related Deaths and Complications in Women With Vascular Ehlers-Danlos Syndrome," by Murray and colleagues ${ }^{4}$ (no relation to me). These articles add some of the essential details required to improve the clinical image in the manner needed in order to inform the care of patients with pathogenic variants in COL3A1.

The relative rarity of COL3A1 mutations in the human population, with an estimated prevalence of about 1 in 200,000 people, ${ }^{5}$ highlights one of the challenges in HDCG; that is, understanding the details surrounding a rare event in enough detail to then apply that understanding in the next observation of the same or a similar event. The work published here provides specific details about vEDS and helps provide us a model for developing additional information for the type of HDCG that we will need in order to deliver on the promise of 21 stcentury genomic medicine.

In the article by Pepin et al., ${ }^{3}$ a large cohort of individuals with COL3A1 mutations were clinically followed, and important genotype-phenotype correlations were examined. Because of the size of this cohort, detailed observations could be made and reported regarding the effects of sex and specific amino acid substitutions on survival. These data will help to relieve some of the uncertainty regarding prognosis that clinicians and patients face, especially at the time of diagnosis. In addition, an understanding of the allelic heterogeneity of this condition will ultimately allow for better-informed decision making about surgical and other interventions.

In the second article, Murray et al. ${ }^{4}$ track the outcomes associated with pregnancy in vEDS. Clinical guidance for informed choices by women in this arena is incredibly difficult and will likely remain so. However, with these data, the job of clinicians and the choices of women are informed by a more detailed understanding regarding the risks of morbidity and mortality associated with pregnancy. In addition to this work, I think that there is reason to be hopeful that, as genomic screening becomes more common in the decades ahead, the terribly unfortunate scenario of new vEDS diagnosis coincident with complications may become less common; this will, of course, allow for better application of what is known about this disease at this critical period in life.

Together, these articles help to highlight the tremendous contribution of Peter Byers and his group toward understanding the details of vEDS and other connective tissue diseases. ${ }^{6}$ In this body of work, a picture of what HDCG will look like starts to come into view, not only for vEDS but also for osteogenesis imperfecta and other related diseases. ${ }^{78}$ Like this body of work, HDCG will build on detailed understanding of the gene product, the range of human mutations that occur, the range of clinical expressivity that exists, and the detailed understanding that comes from the aggregation and analysis of massive amounts of clinical data that have been curated to the level of specific variants (or categories of variants) to provide the genomic pixel,

${ }^{1}$ Genomic Medicine Institute, Geisinger Health System, Danville, Pennsylvania, USA. Correspondence: Michael F. Murray (mfmurray1@geisinger.edu) 
that is, the genotype-phenotype correlation at the level of the sequence variant.

As the total clinical genomic data set increases, we will continue to depend on the masterful aggregation of genotypephenotype correlations made by research groups who have the interest and capacity to independently carry this out. We will also increasingly require rich shared resources, such as the National Center for Biotechnology Information's ClinVar, as the gathering place for clinicians and scientists who can perhaps contribute a single piece of the puzzle. ${ }^{9}$ That is, where caring providers can contribute small data sets consisting of individual or family observations of genotype-phenotype correlations, while others, including teams of bioinformaticists and those with expertise in the relevant biologic mechanisms, can carry out the detailed analysis of aggregate data sets to inform patient care.

Remarkably, we are now at a time when a brand new HDTV can be purchased in 2014 for the same price or less than a stateof-the-art color TV cost in 1954-and HDTV is therefore significantly less expensive. ${ }^{10} \mathrm{I}$ am hopeful that the day will come in the next 60 years when affordable HDCG will be readily available to the public. That HD product will provide a clear picture of sequence-level genotype-phenotype correlations in the context of better understood modifying factors, such as epigenetics and environmental factors.

\section{DISCLOSURE}

The author declares no conflict of interest.

\section{REFERENCES}

1. Wikipedia. High Definition Television (HDTV). http://www.en.wikipedia.org/ wiki/High-definition_television. Accessed 3 June 2014.

2. Wikipedia. Pixel. http://www.en.wikipedia.org/wiki/Pixel. Accessed 3 June 2014

3. Pepin MG, Schwarze U, Rice KM, Lui M, Leistritz D, Byers PH. Survival is affected by mutation type and molecular mechanism in vascular Ehlers-Danlos syndrome (EDS type IV). Genet Med 2014;16:881-888 (this issue).

4. Murray ML, Pepin M, Peterson SE, Byers PH. Pregnancy-related deaths and complications in women with vascular Ehlers-Danlos syndrome. Genet Med 2014;16:874-880 (this issue).

5. Pepin MG, Byers PH. Ehlers-Danlos syndrome type IV. In: Pagon RA, Adam MP, Ardinger $\mathrm{HH}$, et al (eds). GeneReviews ${ }^{\circledR}$ [Internet]. University of Washington, Seattle, WA, 1993-2014. PMID: 20301667.

6. Pace JM, Atkinson M, Willing MC, Wallis G, Byers PH. Deletions and duplications of Gly-Xaa-Yaa triplet repeats in the triple helical domains of type I collagen chains disrupt helix formation and result in several types of osteogenesis imperfecta. Hum Mutat 2001;18:319-326.

7. Marini JC, Forlino A, Cabral WA, et al. Consortium for osteogenesis imperfecta mutations in the helical domain of type I collagen: regions rich in lethal mutations align with collagen binding sites for integrins and proteoglycans. Hum Mutat 2007;28:209-221.

8. Byers PH, Murray ML. Heritable collagen disorders: the paradigm of the EhlersDanlos syndrome. J Invest Dermato/ 2012;132(E1):E6-11.

9. Landrum MJ, Lee JM, Riley GR, et al. ClinVar: public archive of relationships among sequence variation and human phenotype. Nucleic Acids Res 2014;42(Database issue):D980-D985.

10. Wikipedia. Color Television. http://www.en.wikipedia.org/wiki/Color_television. Accessed 3 June 2014. 